\title{
Experimental Evolution of Escherichia coli Persister Levels Using Cyclic Antibiotic Treatments
}

\author{
Bram Van den Bergh, Joran E. Michiels*, and Jan Michiels
}

\begin{abstract}
Persister cells are difficult to study owing to their transient nature and their usually small number in bacterial populations. In the past, numerous attempts have been made to elucidate persistence mechanisms. However, because of the challenges involved in studying persisters and the clear redundancy in mechanisms underlying their generation, our knowledge of molecular pathways to persistence remains incomplete. Here, we describe how to use experimental evolution with cyclic antibiotic treatments to generate mutants with an increased persister level in stationary phase, ranging from the initial ancestral level up to $100 \%$. This method will help to unravel molecular pathways to persistence, and opens up a myriad of new possibilities in persister research, such as the convenient study of nearly pure persister cultures and the possibility to investigate the role of time and environmental aspects in the evolution of persistence.
\end{abstract}

Keywords: Experimental evolution, Persister mutants, Adaptation, Cyclic treatments, Escherichia coli, Bet-hedging, Aminoglycosides

\section{Introduction}

Persistence is a bet-hedging strategy that allows microbial populations to avoid eradication by otherwise lethal antibiotic treatments through the formation of antibiotic tolerant variants (usually 0.0001-0.1\% of the total population) as a first line of defense $[1,2]$. Persisters do not divide during antibiotic treatment and can only resume growth after reverting to the antibiotic-sensitive non-persister phenotype when the antibiotic concentration has dropped, distinguishing them from resistant cells [3]. As such they can avoid clinical detection while causing therapy failure and the resurgence of infection, especially given their presence in biofilms where they are shielded from immune components [4].

\footnotetext{
*Authors contributed equally.
} 
Several groups have searched for persistence genes and mechanisms using classical approaches like screening mutant libraries [5-13], overexpression libraries $[14,15]$, and transcriptome studies on isolated populations enriched for persisters [16-19]. Although progress has been made and several genes and mechanisms have been reported recently [20,21], many questions remain [22]. The usefulness of genetic screens is inherently limited for the study of complex traits such as persistence, and the rare and transient character of the persister phenotype makes transcriptomic analysis impossible without cumbersome enrichment protocols.

We propose experimental evolution as an alternative approach to study persistence for the following reasons. First, experimental evolution is a powerful tool for studying complex, redundant phenotypes [23]. Second, there is no bias in the type of mutations that can be found (e.g. gain-of-function mutations and mutations in regulatory domains or essential genes) [24]. Third, it has already been shown that persister levels are evolvable, both in vivo $[25,26]$ and in vitro [27]. Here, we demonstrate a method to evolve Escherichia coli populations and generate mutants producing a range of persister levels up to $100 \%$ in stationary phase.

\section{Materials}

\subsection{General Material}

Prepare all solutions and media using deionized water and store at room temperature unless stated otherwise. Use E. coli strains carrying unique markers as ancestor. For example, we use E. coli BW25993 carrying different fluorescent markers [28] (see Note 1). Always follow safety instructions when handling hazardous goods or equipment. When disposing waste, please follow local regulations and make sure biological waste is sterilized properly in advance.

1. Antibiotic stock solution: amikacin $50 \mathrm{mg} / \mathrm{ml}$ (see Note 2 ). Weigh $50 \mathrm{mg}$ of amikacin powder stored at $4{ }^{\circ} \mathrm{C}$ and add sterile ultrapure water (resistivity of $18.2 \mathrm{M} \Omega / \mathrm{cm}$ at $25^{\circ} \mathrm{C}$ ) to a final volume of $1 \mathrm{ml}$. Filter sterilize $(0.22 \mu \mathrm{m})$ and store aliquots immediately at $-20^{\circ} \mathrm{C}($ see Note 3$)$.

2. Mueller Hinton Broth (MHB): follow the instructions of the supplier of premixed MHB powder (see Note 4).

3. Lysogeny Broth (LB) agar: weigh $10 \mathrm{~g}$ tryptone, $10 \mathrm{~g} \mathrm{NaCl}$, $5 \mathrm{~g}$ yeast extract and $15 \mathrm{~g}$ agar and add water to a final volume of 11 before autoclaving. Keep the sterile broth on at $50-60{ }^{\circ} \mathrm{C}$ for maximum 2 days before pouring. Store plates at $4{ }^{\circ} \mathrm{C}$ for up to 1 month before use.

4. Magnesium sulfate solution $\left(\mathrm{MgSO}_{4}, 10 \mathrm{mM}\right)$ : weigh $2.46 \mathrm{~g}$ of $\mathrm{MgSO}_{4} \cdot 7 \mathrm{H}_{2} \mathrm{O}$ and add water to a final volume of 11 before autoclaving. 


\subsection{Determination of Antibiotic-Related Characteristics of Ancestral Strain}

\subsection{Evolution Experiment}

5. Sterile glass test tubes and Erlenmeyer flasks of $250 \mathrm{ml}$ (see Note 5).

6. Sterile plastic tubes of 15 and $50 \mathrm{ml}$, suitable for centrifugation.

7. Microcentrifuge capable of $4000 \times \mathfrak{g}$.

8. Centrifuge for $50 \mathrm{ml}$ tubes capable of $4000 \times \mathfrak{g}$.

9. Incubator at $37^{\circ} \mathrm{C}$, fitted with trays for test tubes, microplates and Erlenmeyer flasks, capable of shaking at $200 \mathrm{rpm}$ ( see Note 6).

10. Spiral plater and automated colony counter or alternatively, sterile glass beads for manual plating and counting.

1. MHB agar plates containing different antibiotic concentrations: add $15 \mathrm{~g}$ of agar to 11 of $\mathrm{MHB}$ before autoclaving. Keep the sterile broth on at $50-60{ }^{\circ} \mathrm{C}$ for maximum 2 days before pouring. Mix the sterile broth with amounts of antibiotic stock solution to reach the desired concentrations before pouring. Store $\mathrm{MHB}$ plates containing antibiotics at $4{ }^{\circ} \mathrm{C}$ for up to 1 week before use [29].

2. Sterile 96-well microplate with lid and breathable sterile adhesive seals.

3. 96-well $\mathrm{OD}_{595 \mathrm{~nm}}$ reader.

1. Micropipette tips with filters.

2. Disposable gloves.

3. Disinfection ethanol $70 \%(\mathrm{v} / \mathrm{v})$ : mix 30 parts of water with 70 parts of denatured ethanol.

4. Cryoprotectant: glycerol $50 \%(\mathrm{v} / \mathrm{v})$ : $\operatorname{mix} 50$ parts of water with 50 parts of glycerol.

5. Polypropylene tubes suitable for $-80{ }^{\circ} \mathrm{C}$ preservation (cryotube).

6. Fluorescence microscope (see Note 7).

\section{Methods}

\subsection{Characterization of Antibiotic-Related Characteristics of Ancestral Strain}

Perform all handlings at room temperature and work under sterile conditions. Incubation is carried out at $37^{\circ} \mathrm{C}$, shaking at $200 \mathrm{rpm}$ for liquid cultures. Centrifugation steps are performed at $4000 \times \mathfrak{g}$.

The minimum inhibitory concentration (MIC), the minimum bactericidal concentration (MBC), the mutant prevention concentration (MPC) and the antibiotic concentration and treatment duration needed to reach the persister plateau must be determined for the ancestor before the start of the evolution experiment, and for evolved populations and/or clones afterwards (see Notes 8 and 9). Subheadings 3.1 .1 and 3.1 .2 are adaptations from previously described methods [30-32]. 
3.1.1 MIC and MBC Determination
3.1.2 MPC Determination
1. Inoculate the ancestral strain ( see Notes $\mathbf{1}$ and $\mathbf{9}$ ) in a test tube containing $5 \mathrm{ml}$ of $\mathrm{MHB}$ and incubate overnight.

2. Adjust the optical density of the overnight culture at $595 \mathrm{~nm}$ $\left(\mathrm{OD}_{595 \mathrm{~nm}}\right)$ to 0.5 by centrifugation in a microcentrifuge ( $5 \mathrm{~min}$ ) and resuspending and diluting in $\mathrm{MHB}$.

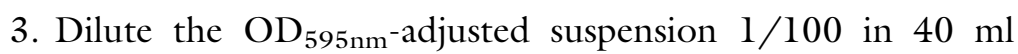
MHB and mix well. Add antibiotic to $10 \mathrm{ml}$ of this inoculum to a final concentration of two times the highest concentration that you want to assess ( see Note 10).

4. Add $150 \mu \mathrm{l}$ of the remaining $30 \mathrm{ml}$ of bacterial inoculum from step 3 to the wells of a sterile microplate. Use three rows as technical replicates per antibiotic per clone and leave column 12 blank. Make also an appropriate tenfold serial dilution series in $\mathrm{MgSO}_{4}$ solution $(10 \mathrm{mM})$ of the remaining inoculum and plate out on LB agar. Count colonies after overnight incubation to determine the CFU per $\mathrm{ml}$.

5. Add $150 \mu \mathrm{l}$ of MHB to column 12 as negative control.

6. Make a twofold serial dilution series of the antibiotic: add $150 \mu \mathrm{l}$ of the inoculum with antibiotic to the wells of column 1 , mix, and transfer $150 \mu \mathrm{l}$ to the next column and repeat for each column until column 10. Mix column 10 and remove $150 \mu \mathrm{l}$. Leave column 11 without antibiotics as a positive control. Cover the microplate with a breathable seal and plastic lid, and incubate for 16-20 h.

7. Measure the $\mathrm{OD}_{595 \mathrm{~nm}}$ for each well in a multireader. Verify the positive and negative controls for adequate microbial growth and medium sterility. The MIC is the lowest concentration inhibiting visible growth.

8. Make appropriate tenfold serial dilutions in $\mathrm{MgSO}_{4}(10 \mathrm{mM})$ of the wells containing antibiotic above the MIC (thus showing no visible growth) and plate out on LB agar. Count colonies after overnight incubation to determine the CFU per $\mathrm{ml}$ and compare with CFU per $\mathrm{ml}$ of the inoculum determined in step 4. The MBC is the lowest concentration capable of killing $99.9 \%$ of cells.

1. Inoculate the ancestral strain (see Notes $\mathbf{1}$ and $\mathbf{9}$ ) in a test tube containing $5 \mathrm{ml}$ of $\mathrm{MHB}$ and incubate overnight.

2. Dilute the overnight culture $1 / 100$ in a flask containing $100 \mathrm{ml}$ of $\mathrm{MHB}$ and incubate overnight.

3. Concentrate the overnight culture tenfold: centrifuge $50 \mathrm{ml}$ of the overnight culture $(25 \mathrm{~min})$, discard the supernatant and resuspend the cell pellet in $5 \mathrm{ml}$ of $\mathrm{MgSO}_{4}$ solution $(10 \mathrm{mM})$.

4. Plate out $>10^{10}$ cells on $\mathrm{MHB}$ plates containing a twofold serial dilution range of antibiotic (see Note 10). We use five 


\subsubsection{Construction of Killing Curves in Function of Antibiotic Concentration and Treatment Duration to Determine the Persister Plateau}

plates per antibiotic concentration and plate out $200 \mu$ lof the cell suspension prepared in step $\mathbf{3}$ on each plate. Incubate for $48 \mathrm{~h}$ to allow detection of colonies arising from resistant mutants.

5. The MPC is the lowest concentration inhibiting growth of resistant mutants.

1. Inoculate the ancestral strain (see Notes $\mathbf{1}$ and $\mathbf{9}$ ) in a test tube containing $5 \mathrm{ml}$ of $\mathrm{MHB}$ and incubate overnight.

2. Dilute the overnight culture $1 / 100$ in a flask containing $100 \mathrm{ml}$ of $\mathrm{MHB}$ and incubate overnight.

3. Transfer samples of $1 \mathrm{ml}$ of the overnight culture to test tubes and incubate in the presence of a range of antibiotic concentrations for $5 \mathrm{~h}$. Alternatively, samples can be treated with a fixed antibiotic concentration for various treatment durations to determine killing curves in function of time (see Note 10).

4. Make an appropriate tenfold serial dilution series of the overnight culture in $\mathrm{MgSO}_{4}$ solution $(10 \mathrm{mM})$ and plate out on LB agar. Count colonies after overnight incubation to determine the initial cell number.

5. After antibiotic treatment, spin the samples down in a microcentrifuge ( $5 \mathrm{~min}$ ), wash once with $\mathrm{MgSO}_{4}$ solution $(10 \mathrm{mM})$ to remove the antibiotic, make appropriate tenfold serial dilutions in $\mathrm{MgSO}_{4}$ solution (10 mM) and plate out on LB agar plates. Count colonies after $48 \mathrm{~h}$ of incubation to determine the number of surviving cells ( see Note 11).

6. Plot the number of surviving cells in function of antibiotic concentration or treatment duration to determine the conditions needed to reach the persister plateau (see Note 8) (Fig. 1).

Since evolution experiments often require many days of continuous lab work and many factors can confound the outcome of the experiment (e.g. contaminations, timing of growth and treatment periods, accidental events like power breakdown or glassware breakage and chance resulting in genetic drift), we want to stress the importance of material preparations, thoughtful experimental set-up and careful handling of evolving populations ( see Notes $\mathbf{3}-\mathbf{5}$, 7, 9 and 12-19) to improve replicability and increase work efficiency.

To start the evolution experiment:

1. Inoculate separate single colonies in test tubes containing $5 \mathrm{ml}$ of $\mathrm{MHB}$ and incubate overnight. One colony is used as the founder of each population (see Notes 12 and 13).

2. Dilute the overnight cultures $1 / 100$ in flasks containing $100 \mathrm{ml}$ of $\mathrm{MHB}$ and incubate overnight (see Note 14). 


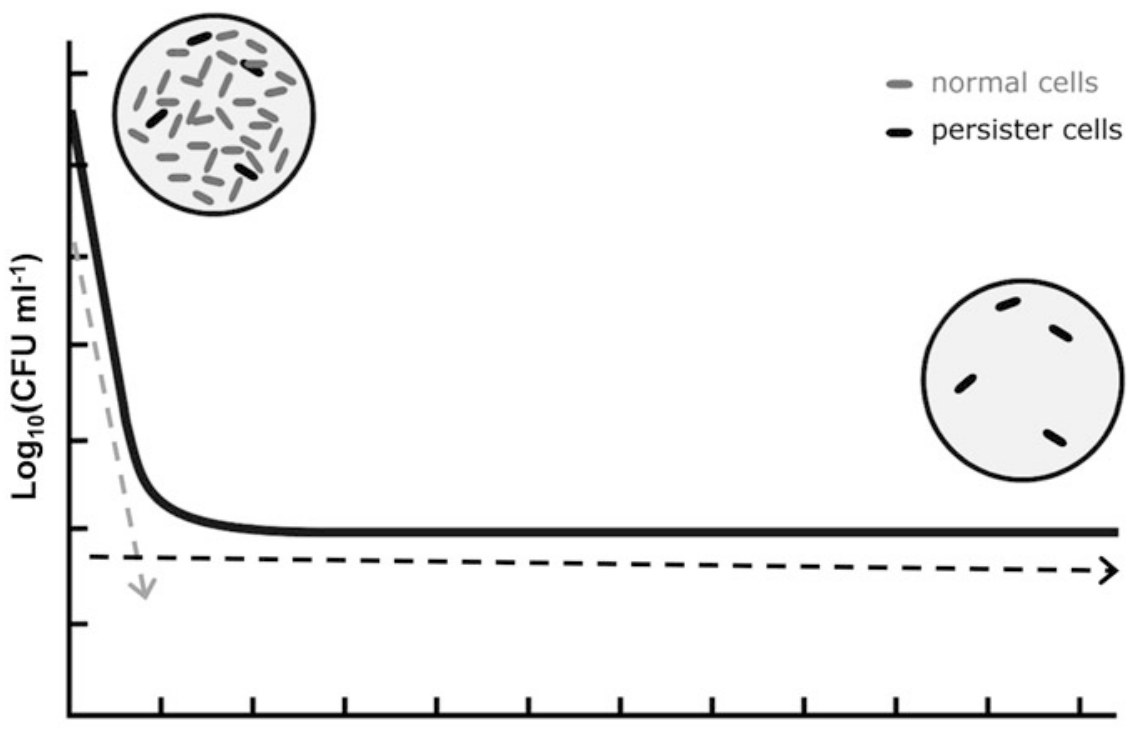

[antibiotic] or treatment time

Fig. 1 Killing curve in function of treatment duration or antibiotic concentration reveals a persister plateau. Initially, the number of surviving cells decreases rapidly with increasing antibiotic concentration or treatment duration. However, at a certain point a plateau is reached where a further increase in concentration or treatment duration only slightly increases killing. At this point, all normal cells are killed, and only highly tolerant persister cells remain. This biphasic pattern is a result of the initial fast killing of normal cells (gray) and the increased survival of persisters (black) at longer treatment times or higher antibiotic concentrations. Antibiotic concentration and treatment time should be chosen to fall within the range sufficient to reach the persister plateau and ideally in a range where the surviving fraction is minimally influenced by small experimental fluctuations. Reproduced and adapted with permission from [20]

3. Depending on the desired treatment frequency, days with treatment should be alternated with days without treatment ( see Note 15). If the overnight culture (resulting from steps 2 , 6 or 8 ) needs to be treated, continue with step 4 . If not, move on to step 8. In case you want to end the experiment, proceed to step 9 ( see Note 16).

On days with treatment:

4. Make appropriate tenfold serial dilutions of the overnight cultures in $\mathrm{MgSO}_{4}(10 \mathrm{mM})$, plate out on $\mathrm{LB}$ agar and incubate overnight to determine the total CFU per $\mathrm{ml}$.

5 . Treat a $2 \mathrm{ml}$ sample $(2 \times 1 \mathrm{ml})$ of each overnight culture with antibiotic. Use a concentration and treatment duration according to the results obtained in Subheading 3.1 ( see Note 8).

6. After antibiotic treatment, spin down the samples in a microcentrifuge ( $5 \mathrm{~min}$ ) and wash three times with $\mathrm{MgSO}_{4}$ solution $(10 \mathrm{mM})$ to remove the antibiotic. Dilute each sample $1 / 100$ in flasks containing $100 \mathrm{ml}$ of $\mathrm{MHB}$ and incubate overnight to 
start another cycle of growth (see Note 14). Make appropriate tenfold serial dilutions of the remaining of the washed samples in $\mathrm{MgSO}_{4}$ solution $(10 \mathrm{mM})$, plate out on LB agar and incubate for $48 \mathrm{~h}$ to determine CFU per $\mathrm{ml}$ of surviving cells ( see Note 11). The ratio of CFU per $\mathrm{ml}$ of surviving cells to CFU per $\mathrm{ml}$ of total cells is defined as the persister fraction and should be monitored at regular time intervals throughout the experiment.

7. Irrespective of the desired treatment frequency, pellet $50 \mathrm{ml}$ of the overnight cultures resulting from step 6 by centrifugation (25 min). Resuspend the pellet in $\mathrm{MgSO}_{4}$ solution $(10 \mathrm{mM})$ to a final volume of $1 \mathrm{ml}$, add $1 \mathrm{ml}$ of glycerol $(50 \%, \mathrm{v} / \mathrm{v})$ and store immediately at $-80{ }^{\circ} \mathrm{C}($ see Note 16). Go to step 3 .

On days without treatment:

8. Dilute the overnight cultures from step 6 or step $81 / 1,000,000$ in flasks containing $100 \mathrm{ml}$ of MHB and incubate overnight to start another cycle of growth ( see Notes 14 and 17). Go to step 3.

To end or interrupt the experiment (see Note 16):

9. Check every population for contaminations. We use different fluorescent markers to check for possible contamination using fluorescence microscopy (see Notes 1 and 18).

10. Cryopreserve the overnight cultures as described in step 7.

11. Plate out the overnight cultures as described in step 4.

12. Isolate clonal endpoints: pick single colonies and use these to inoculate test tubes containing $5 \mathrm{ml}$ of MHB (see Note 19). Incubate overnight.

13. Cryopreserve the clones at $-80{ }^{\circ} \mathrm{C}$ : $\mathrm{mix} \mathrm{l} \mathrm{ml}$ of overnight culture with $1 \mathrm{ml}$ of glycerol $(50 \%, \mathrm{v} / \mathrm{v})$.

14. Analyze the antibiotic-related characteristics of the clones and/ or the populations according to Subheading 3.1 (see Notes 8 and 9 ).

\section{Notes}

1. We have used numerous E. coli lab strains with different genetic backgrounds but also pathogenic strains and even other species (e.g. Salmonella Typhimurium) to evolve high persister levels. Ideally, the selected strain should allow detection of (cross)contamination (e.g. different fluorescent markers, auxotrophic markers), especially in initial experiments. Any extra information (e.g. genomic sequence) can also be taken into account when choosing an ancestral strain. Evidently, the strain should be sensitive to the used antibiotic. 
2. We have confirmed our method with other aminoglycosides (kanamycin, tobramycin, and gentamicin). The concentration of the stock solution may be adapted in function of the desired final concentration. When shifting from formulation (e.g. hydrate, sulfates) or supplier, take into account the possible potency difference to achieve similar concentrations.

3. When using antibiotic stock solutions over a long time period (e.g. during a long-term evolution experiment), minimize freeze-thaw cycles of aliquots and renew antibiotic stocks frequently to avoid attenuation of antibiotic efficacy. Amikacin should not be stored for longer than 1 month at $-20{ }^{\circ} \mathrm{C}$ [29]. Therefore, adjust the amount of antibiotic stock solution and volume of the aliquots to the expected needs.

4. Using premixed $\mathrm{MHB}$ formulas may enhance reproducibility. Since company formulas may differ, it is recommended to stick to one supplier and manage MHB stocks well to tackle accidental events in advance (e.g. problems with deliveries or differences between batches). Alternatively, MHB can be prepared using $2 \mathrm{~g} / 1$ beef Extract powder, $17.5 \mathrm{~g} / 1$ acid digest of casein, and $1.5 \mathrm{~g} / 1$ starch. In some cases (depending on the organism and antibiotic of interest [30]) $\mathrm{Ca}^{2+}$ and $\mathrm{Mg}^{2+}$ content of $\mathrm{MHB}$ needs to be adjusted to final concentrations of $20-25 \mathrm{mg} / \mathrm{l}$ and 10-12.5 mg/1, respectively. Transfer MHB to other recipients (test tubes, Erlenmeyer flasks) before autoclaving and under continuous mixing or after gentle heating to dissolve it completely. When preparing MHB medium for the evolution experiment itself, please reserve sufficient $\mathrm{MHB}$ powder of the same batch for the entire experiment, never store the medium after autoclaving for longer than a week, store at room temperature and away from light and never autoclave twice.

5. Make sure to provide at least three times the number of populations in Erlenmeyer flasks when starting an evolution experiment. Depending on the turnover of your institution's glassware cleaning and the length of the experiment, four or five times the number of populations in flasks may be required.

6. For comparison between parallel evolutionary lines and to compare between independent phenotyping experiments, it is best stick to one incubator or use incubators with identical properties (e.g. shaking amplitude). In our case, $200 \mathrm{rpm}$ equals to $0.11 \mathrm{~g}$.

7. Depending on the unique markers present in the ancestral E. coli strains, other means may be needed to check for contamination.

8. These parameters need to be determined at least in biological triplicate. We recommend the antibiotic concentration in the evolution experiment to be at least ten times as high as the MIC 
and MBC values, and well above the MPC. The antibiotic concentration and treatment duration also need to be sufficiently high to reach the persister plateau. In our case, antibiotic treatments using amikacin concentrations of minimum $50 \mu \mathrm{g} / \mathrm{ml}$ and maximum $400 \mu \mathrm{g} / \mathrm{ml}$ for $5 \mathrm{~h}$ were successfully used in the evolutionary set-up. After the evolution experiment, Subheading 3.1 can be used to assess evolution of genuine persistence, i.e. increased survival with no accompanying changes in MIC value, and to assess evolution of the other antibiotic-related characteristics.

9. If using evolved populations, it is best to limit the amount of growth cycles to reduce further evolution and change in population compositions. Therefore, for populations, Subheading 3.1 could be performed during the evolution experiment. Alternatively and more practically, frozen stocks can also be used as starting point. We advise to thaw these stocks completely and dilute them $1 / 100$ in $\mathrm{MHB}$ medium to allow for one overnight growth cycle to stationary phase to obtain sufficient starting material for Subheading 3.1.

10. MIC, MBC, MPC and concentration and treatment durations needed to reach a persister plateau can vary between different species and even between $E$. coli strains. If no prior information is available, we advise starting with a broad range and/or few concentrations. In order to determine the concentrations and treatment durations needed to reach a persister plateau, we always start with optimizing the antibiotic concentration using a fixed and practical treatment time (e.g. 5 h, which leaves enough time to complete growth to stationary phase during a total cycle of $24 \mathrm{~h}$ ). Subsequently, the persister plateau should be verified in function of time.

11. Since persister cells have longer lag times than normal cells $[2,27]$, we incubate for $48 \mathrm{~h}$ instead of $24 \mathrm{~h}$ when plating out after treatment to ensure colony formation of most persister cells.

12. Founding each population from a different colony can prevent compromised results due to the influence of random mutations that might be present in some initial clones. We also advise to replica plate these colonies at the start of the experiment on LB agar and cryopreserve part of the first overnight cultures from step 1 at $-80{ }^{\circ} \mathrm{C}$, e.g. by mixing $1 \mathrm{ml}$ of culture with $1 \mathrm{ml}$ of glycerol $(50 \%, \mathrm{v} / \mathrm{v})$ to be able to track back particular observations to the founding colony. Depending on the objective of the experiment, the number of replicate populations can be increased or decreased. For example, for a try-out experiment or when the goal is to obtain a high persister mutant for practical reasons in a follow-up study, 1-3 replicates might suffice. 
In other cases, for example when the goal is to follow evolution over longer timescales or to study the dynamics of the evolutionary process, it is advised to increase the number of replicate populations to reduce the effect of chance and increase the study's resolution. However, keep in mind that each increase in number of replicates also implies increased work load, materials, and infrastructure demands. If high numbers of replicates are nevertheless necessary, good preparation becomes especially essential (see Subheading 2).

13. If you have access to isogenic tagged strains ( encourage to use them as founders for each population, especially in initial experiments. If you have more populations than uniquely marked strains, we suggest to alternately handle populations with contrasting markers. In this way, crosscontamination may be identified more easily. To limit contamination of any kind, we advise to wear gloves, disinfect gloves and materials between handling different populations, use micropipette tips with filters when handling evolving populations and exclusively use freshly autoclaved solutions of glycerol $(50 \%, \mathrm{v} / \mathrm{v})$ and $\mathrm{MgSO}_{4}$ solution $(10 \mathrm{mM})$. Never open two flasks or recipients containing evolving populations at the same time.

14. To avoid accidental events that might result in the premature ending of the experiment, we advise storing stationary phase cultures on days with and without treatment at $4{ }^{\circ} \mathrm{C}$ as backup until populations have reached the next stationary phase.

15. Although treatment frequency is generally expressed as "once every x days", identical treatment frequencies can be obtained in different ways. For example, when the treatment frequency is once every 8 days, the set-up can start with a treatment followed by 7 dilution steps, start with 7 dilution steps and end with a treatment, or any situation in between. For uniformity, we always start with a treatment step followed by dilution steps in any given setup. On days without treatment, persister levels can still be assayed if necessary, analogous to steps 4-6 but without inoculating the next cycle of evolution with persister cells.

16. At the last day of the evolution experiment, we once again assay persister levels by repeating steps 4-6 and freeze the resulting overnight culture like in step 7. In this way, the evolution experiment can always be restarted if necessary. Although we advise to perform the experiment without interruption, this allows to pause the experiment if needed. It is possible and sometimes advisable to store samples at $-80{ }^{\circ} \mathrm{C}$ more frequently as backup and cryopreserved evolution library. 
As a minimum, we advise to cryopreserve overnight cultures after every treatment cycle. In this way, if you need to start over, the populations can be inoculated from completely thawed cryopreserved backups with a dilution factor similar to step $\mathbf{8}$. Furthermore, the populations then have at least one growth cycle to adjust from $-80{ }^{\circ} \mathrm{C}$ conditions before treatment starts. In case the treatment frequency is once every day, persister cells at the end of the experiment (see Note 14), or at intermediate points, can be cryopreserved at $-80{ }^{\circ} \mathrm{C}$ after removal of the antibiotic as in step 6.

17. On days without treatment, we dilute samples $1 / 1,000,000$ to mimic the bottleneck populations experience on treatment days. Depending on the initial persister level (in our case $10^{-3}-10^{-4}$ ), we advise to adjust this dilution factor. However, keep in mind that strong bottlenecks increase the effect of genetic drift, especially when initial persister levels are low. Furthermore, when using lower treatment frequencies, the selection pressure is not as strong, increasing the influence of drift [33].

18. We advise to check for contamination also at intermediate points during the experiment and definitely when storing samples to pause the experiment. Since the presence of a small number of non-fluorescent cells within a whole population might be difficult to observe, repeat the contamination check on the randomly selected clones.

19. The number of clonal endpoints to isolate from each population is somewhat arbitrary, but again depends on the goal of the experiment. If treatment frequency is low, we would recommend to pick at least five clones per population since variability within populations and between replicate populations might be high. If treatment frequency is high, the number of clones (and the number of replicate populations to isolate clones from) may be decreased.

\section{Acknowledgements}

The authors are fellows of the Research Foundation-Flanders (FWO) and the Agency for Innovation by Science and Technology (IWT). The research was further supported by grants from the KU Leuven Research Council (PF/10/010; IDO/09/010) and the IAP-BELSPO initiative. 


\section{References}

1. Cohen NR, Lobritz MA, Collins JJ (2013) Microbial persistence and the road to drug resistance. Cell Host Microbe 13:632-642

2. Balaban NQ, Merrin J, Chait R et al (2004) Bacterial persistence as a phenotypic switch. Science 305:1622-1625

3. Lewis K (2010) Persister cells. Annu Rev Microbiol 64:357-372

4. Fauvart M, De Groote VN, Michiels J (2011) Role of persister cells in chronic infections: clinical relevance and perspectives on antipersister therapies. J Med Microbiol 60:699-709

5. Manuel J, Zhanel GG, de Kievit T (2010) Cadaverine suppresses persistence to carboxypenicillins in Pseudomonas aeruginosa PAOl. Antimicrob Agents Chemother 54:5173-5179

6. Li Y, Zhang Y (2007) PhoU is a persistence switch involved in persister formation and tolerance to multiple antibiotics and stresses in Escherichia coli. Antimicrob Agents Chemother 51:2092-2099

7. Girgis HS, Harris K, Tavazoie S (2012) Large mutational target size for rapid emergence of bacterial persistence. Proc Natl Acad Sci U S A 109:12740-12745

8. De Groote VN, Verstraeten N, Fauvart $M$ et al (2009) Novel persistence genes in Pseudomonas aeruginosa identified by high-throughput screening. FEMS Microbiol Lett 297:73-79

9. Leung V, Levesque CM, Lévesque CM (2012) A stress-inducible quorum-sensing peptide mediates the formation of persister cells with noninherited multidrug tolerance. J Bacteriol 194:2265-2274

10. Hu Y, Coates ARM (2005) Transposon mutagenesis identifies genes which control antimicrobial drug tolerance in stationary-phase Escherichia coli. FEMS Microbiol Lett 243:117-124

11. Dhar N, McKinney JD (2010) Mycobacterium tuberculosis persistence mutants identified by screening in isoniazid-treated mice. Proc Natl Acad Sci USA 107:12275-12280

12. Ma C, Sim S, Shi W et al (2010) Energy production genes $s u c B$ and $u b i F$ are involved in persister survival and tolerance to multiple antibiotics and stresses in Escherichia coli. FEMS Microbiol Lett 303:33-40

13. Hansen S, Lewis K, Vulić M (2008) Role of global regulators and nucleotide metabolism in antibiotic tolerance in Escherichia coli. Antimicrob Agents Chemother 52:2718-2726
14. Spoering AL, Vulic M, Lewis K et al (2006) GlpD and PlsB participate in persister cell formation in Escherichia coli. J Bacteriol 188: 5136-5144

15. Germain E, Castro-Roa D, Zenkin N et al (2013) Molecular mechanism of bacterial persistence by HipA. Mol Cell 52:248-254

16. Shah D, Zhang Z, Khodursky A et al (2006) Persisters: a distinct physiological state of E. coli. BMC Microbiol 6:53

17. Keren I, Minami S, Rubin E et al (2011) Characterization and transcriptome analysis of $\mathrm{Myco}$ bacterium tuberculosis persisters. MBio 2: e00100-e00111

18. Keren I, Shah D, Spoering A et al (2004) Specialized persister cells and the mechanism of multidrug tolerance in Escherichia coli. J Bacteriol 186:8172-8180

19. Van Acker H, Sass A, Bazzini S et al (2013) Biofilm-grown Burkbolderia cepacia complex cells survive antibiotic treatment by avoiding production of reactive oxygen species. PLoS One 8:e58943

20. Kint CI, Verstraeten N, Fauvart M et al (2012) $\mathrm{New}$-found fundamentals of bacterial persistence. Trends Microbiol 20:577-585

21. Amato SM, Fazen CH, Henry TC et al (2014) The role of metabolism in bacterial persistence. Front Microbiol 5:70

22. Balaban NQ, Gerdes K, Lewis K et al (2013) A problem of persistence: still more questions than answers? Nat Rev Microbiol 11:587-591

23. Blaby IK, Lyons BJ, Wroclawska-Hughes E et al (2012) Experimental evolution of a facultative thermophile from a mesophilic ancestor. Appl Environ Microbiol 78:144-155

24. Barrick JE, Yu DS, Yoon SH et al (2009) Genome evolution and adaptation in a longterm experiment with Escherichia coli. Nature 461:1243-1247

25. Lafleur MD, Qi Q, Lewis K (2010) Patients with long-term oral carriage harbor highpersister mutants of Candida albicans. Antimicrob Agents Chemother 54:39-44

26. Mulcahy LR, Burns JL, Lory S et al (2010) Emergence of Pseudomonas aeruginosa strains producing high levels of persister cells in patients with cystic fibrosis. J Bacteriol 192:6191-6199

27. Fridman O, Goldberg A, Ronin I et al (2014) Optimization of lag time underlies antibiotic tolerance in evolved bacterial populations. Nature 513:418-421 
28. Yu J, Xiao J, Ren X et al (2006) Probing gene expression in live cells, one protein molecule at a time. Science 311:1600-1603

29. Andrews JM (2001) Determination of minimum inhibitory concentrations. J Antimicrob Chemother 48(Suppl 1):5-16

30. Wiegand I, Hilpert K, Hancock REW (2008) Agar and broth dilution methods to determine the minimal inhibitory concentration (MIC) of antimicrobial substances. Nat Protoc $3: 163-175$
31. Liebens V, Defraine V, Van der Leyden A et al (2014) A putative de-N-acetylase of the PIG-L superfamily affects fluoroquinolone tolerance in Pseudomonas aeruginosa. Pathog Dis 71:39-54

32. Drlica K (2003) The mutant selection window and antimicrobial resistance. J Antimicrob Chemother 52:11-17

33. Barrick JE, Lenski RE (2013) Genome dynamics during experimental evolution. Nat Rev Genet 14:827-839 